\title{
Artistic Characteristics of Foshan Fancy Lantern
}

\author{
Jianfei Li \\ Foshan Huacai Vocational-technical School \\ Foshan, China
}

\begin{abstract}
Foshan fancy lantern has a long history and a profound cultural background, and unique themes, techniques and patterns. This thesis, through the analysis of creative themes, craft characteristics and presentation art of Foshan fancy lantern, shows the magnificent and splendid, dainty and exquisite characteristics of Foshan fancy lantern and the charm full of local characteristics.
\end{abstract}

Keywords-Foshan fancy lantern; artistic characteristics; splendid and magnificent; dainty and exquisite

\section{INTRODUCTION}

Folk fancy lanterns circulated in the Song Dynasty in Guangdong, forming a unique and prosperous vein in Foshan. People call it "decorative lantern". It has the delicate and beautiful characteristics of the South China lantern. Foshan fancy lanterns are mainly made of bamboo strips, iron wire and other materials by hand. After modeling, paste all kinds of silk fabric, lace and color panicle with characteristic Foshan paper-cut patterns or painting patterns. It has the local customs and traditional colors of Foshan. It is the beautiful spectacle of "the most enviable plaice lantern and the lantern with lush flower tree pattern"1. One special characteristic of Foshan fancy lanterns is that they are decorated with paper-cut patterns or mounting patterns on lanterns. The paper-cut formed a silhouette on the bright lantern, forming a profound and moving beauty. The perfect combination of two kinds of art, Foshan paper-cut and Foshan lantern, is also the crystallization of the wisdom of the working people in Foshan.
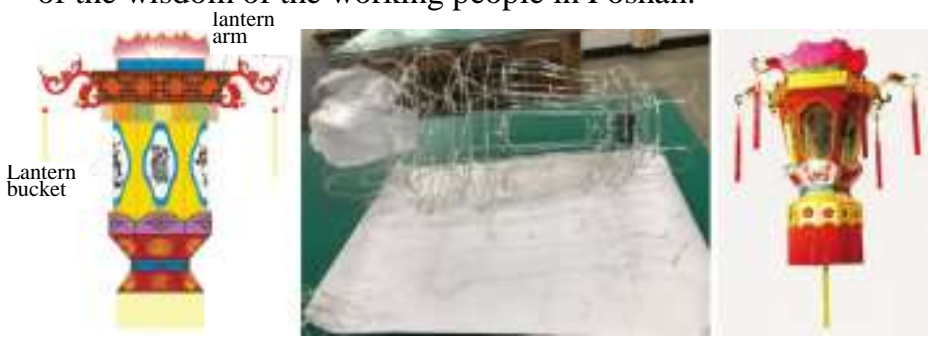

Fig. 1. Creative idea, production implementation and physical effect.

\section{THE CRAFTAND PATTERN OF FOSHAN FANCY LANTERN}

Foshan fancy lanterns are classified in different ways according to different angles. According to the craft and application of Foshan fancy lantern, it is divided into two categories: wire-bundled fancy lantern and special art lantern ${ }^{2}$. Foshan fancy lantern, which is described in this thesis, refers to the wire-bundled line-fit lantern, which is also called wirebundled fancy lantern.

As for wire-bundled fancy lantern, the structure, also called skeleton, is bundled by bamboo strips or wire. In dialect, Foshan fancy lantern is called "lang". Fit silk, paper or other soft materials on the structure, and set lighting device in the interior. It is a kind of bundled lantern. The wire-bundled fancy lantern includes palace lantern, image-imitation lantern, large group lantern and colored lantern. In the processing of wire-bundled fancy lantern, first craftsman designs the contour image of lantern, the connection dimension of entity and detail, selection of materials, cutting, colors, patterns and assembly process. Second, bundle the structure of lantern, which is also called "make lang". The procedure of making lang is the core process of making lantern. Traditionally use bamboo strips or iron wire to bundle the skeleton of lantern by hand. Tie every connection with sticky sandy paper. If make lang for large lantern, based on traditional bundled methods, add engineering mechanics. Originally use sticky sandy paper to bundle fine wire firm. But now replace it with right steel wire and weld it. Replace bamboo strip with rattan to improve the elasticity. Third, it is the line-fit procedure. Add flesh to the structure and form screen of fancy lantern. Use waterproof sunscreen for outdoor lantern. Fourth, it is the decoration procedure. Papercut decoration is an important link in screen decoration, which is also the last stage in making fancy lantern. Set lighting device in the fancy lantern, and motor for revolving scenic lantern. Then a dynamic and static integrated lantern is finished.

Paper-cut art has a long history and cultural heritage. The application of it in Foshan fancy lantern occupies an extremely important position, which may be derived from its own characteristic of Foshan paper-cut. Foshan paper-cut has a long history, and it is closely integrated with the prosperous business culture of Foshan. The ancient town Foshan, the first of the four famous towns, was famous for the handicraft industry in the Ming and Qing Dynasties. It was also the largest distribution center in the south China. Agriculture, handicraft industry and commercial trade were quite developed, which brought up a lot of skilled craftsmen. Once the material conditions for paper cutting are available, Foshan paper cutting

\footnotetext{
Guan Hong: Foshan Fancy Lantern, Guangdong People's Publishing House, 2017, Page 1
}

Guan Hong: Foshan Lantern, Guangdong People's Publishing House, 2017, Page 152 
is created by local skilled craftsmen ${ }^{3}$. There are two categories, cutting and carving, in the production. Use the copper foil and silver foil of local special products, use the techniques of shearing, carving and chiseling, line all kinds of color paper and draw various patterns to form a unique local style of strong color, splendor and magnificence, vigor and boldness. In manufacturing methods, there are three main categories: material paper engraving, writing paper cutting, and pure-color paper cutting. Copper lining, copper writing material and copper chisel material are the most distinctive features. Commonly use copper foil to decorate screen of Foshan lantern, making the lamp shining and elegant. Paper cutting has become one of the special ornaments of Foshan fancy lantern.

\section{A. The Craft and Pattern of Lantern Screen}

The lantern screen is the key decoration part of the lantern, and it is also an important place attracting people's attention. Paper-cuts for fancy lantern screen are often pure-color papercut, lining-color paper-cut, writing-color paper-cut and copper chisel paper-cut. There are four categories to decorate lantern ${ }^{4}$. This kind of decorative paper-cut on the lantern piece does not need to become a complete picture independently. It is also called semi-finished lantern pattern which also has the connotations of good fortune, Ruyi, receiving blessings, eliminating evil and avoiding adversities. For example, there are lotus pattern, Ruyi pattern, cloud head pattern, Taiji pattern, scale pattern, “万”-character pattern, geometric pattern, vortex dot pattern, honeysuckle pattern, tangcao pattern, tangledbranch pattern and so on. These patterns have been evolved from traditional patterns, and have also been influenced by foreign cultures, such as complicated pattern, colored drawing and Rococo style. There is also a key point in cutting the screen paper-cut. That is the decoration of the lantern bucket. The bucket is the ornamental center of each lantern. When designing the lantern modeling, the center position of the lantern is specially emphasized or the lantern bucket of concave and convex sensation is made. Use poetry painting or paper-cut to decorate lantern bucket. If use paper-cut to decorate it, it shall emphasizes the theme of paper-cut patterns. For example, "butterfly loves flower" lantern shall de designed with the theme of butterfly and flower; "fair lady" lantern shall be designed with the theme of beautiful women.

Often use copper foil paper to cut paper-cut for fancy lanterns. Use a knife to carve image and outline on copper foil as copper lining. Then line with color papers on its back according to the design. The lining colors of cooper lining mainly are orange, pink, auramine, mustard yellow, purple, dark green and light blue, and it looks bright and eye-catching. Copper writing material is an art form combining painting and paper cutting. First, use the graver to engrave the outline of the picture on the copper foil, then use glue to adjust the pastel color and draw the picture. The copper chisel material is roughly the same as the copper writing material. Its feature is that the picture or figure outline is chiseled with a special

Yan Mingxia: Discussion on the Origin of Foshan Paper-cut, Journal of South China Agricultural University, 2008, Fourth Issue.

Guan Hong: Foshan Fancy Lantern, Guangdong People's Publishing House, 2017, Page 212. chisel into a small, protruding pearl line. The space does not need to be carved thoroughly. It mainly uses famille rose for painting and show a splendid and colorful image. Colors of copper chisel material mainly are pink, rose red, powder blue and pink white, which are harmonious and dazzling. In order to create best outdoor display effect, screen of fancy lantern is made of copper lining paper-cut of copper foil. Use copperlining paper-cut to decorate fancy lantern to make it beautiful and elegant.

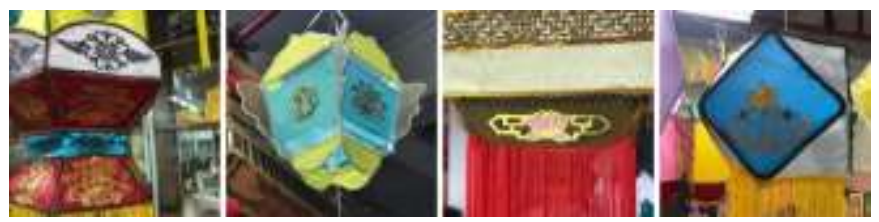

Fig. 2. Decorative pattern of fancy lantern screen.

\section{B. The Craft and Pattern of Lantern Arm}

The lantern arm can improve the decoration effect of fancy lantern, especially the overall effect, so it cannot be neglected. Usually set lantern arm as accessories for fancy lantern to highlight the theme of lantern. The design of lantern arm is especially exquisite. It is shown in the way of Foshan paper cutting. It is more particular about knife cutting and carving method. It is independent, and has the effect of individual integrity. It is an integral part of lantern.

The paper-cut design of lantern arm is often presented by auspicious patterns or bionic images. The design of the lantern arm is according to the theme. It is rigorous in composition, strong in decoration and elegant in appearance. At the same time, use the techniques of cutting, carving, engraving, stenciling and picking, and line with various colors of paper to form a characteristic of strong color and splendor. The lamp arms of the bionic image are the following butterfly-shaped and phoenix-shaped arms. Such patterns are carved with gold foil paper, and line each part with bright red, rose red, green, deep blue and lake blue papers, so that the lanterns seems to be splendid and magnificent.
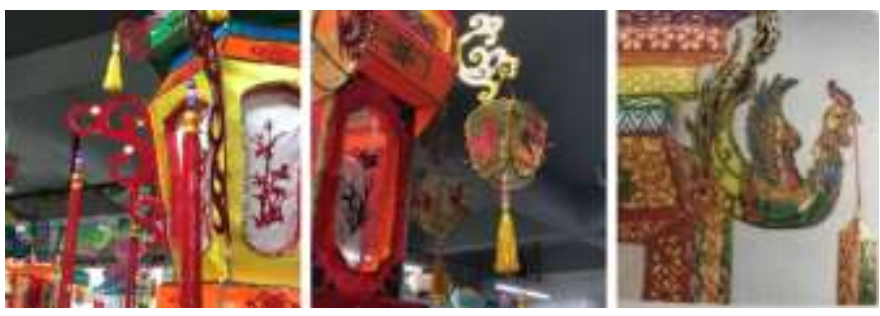

Fig. 3. Effect of lantern arm pattern.

\section{ARTISTIC CHARACTERISTICS OF FOSHAN FANCY LANTERN}

Foshan fancy lantern, known as decorative lantern in Foshan, Guangdong, is one of the main schools of traditional Chinese lantern art, and also the representative of lanterns in south China. The handicraft industry and commerce of Foshan were very developed in the Ming Dynasty, and people's life was stable. The Lantern Festival in the Central Plains, 
appreciating lanterns in the Mid-autumn Festival rose in Foshan, and the wire-bundled craft has come into being ${ }^{5}$. Foshan fancy lantern is commonly called as decorative lantern. It is traditional handicraft of Guangdong Foshan which has the exquisite and beautiful characteristics of southern lanterns in Guangdong Province, and is one of the main schools of traditional lantern art. It includes large lanterns and head lanterns, character story group lanterns, colored dragon and portable lanterns. The whole making process of lantern is operated by hand of craftsmen. Foshan fancy lantern is the representative of Chinese folk lanterns. It is a wonderful work of South of the Five Ridges folk art and an important part of the cultural life of the masses. The lantern is an illumination light in festivals, a light for prayer, a decoration for festivals and a folk art for appreciation. Foshan Parade is also known as Autumn Tamasha. People cruise fancy lanterns, which has the flavor of competitive entertainment. Whose craftwork is the best, whose innovation is the most creative, whose lantern could be praised and recognized by the masses. In order to attract tourists and make the lantern festival appreciative, it motivates folk craftsmen to improve themselves in workmanship. With great originality of folk masters of generations, Foshan fancy lantern is unique in style, exquisite in craft and delicate in making. Especially the combination with Foshan paper cutting makes it more beautiful.

The bundling skill of Foshan fancy lantern is a miracle in the folk art of South of the Five Ridges. In hundreds of years of the history course, it has experienced the stage of rise, prosperity, decline, rebirth, setbacks and rejuvenation. Foshan fancy lantern has the exquisite and beautiful characteristics of fancy lantern in southern China. The bundling craft is leading in China. It is popular with the masses with rich variety, novel style, unique decoration, exquisite craft and magnificent atmosphere. The most characteristic copper-lined paper-cut is an important ornament for fancy lantern. Because of the technology of copper foil as a technological support, copperlined paper cutting has become a connotation of splendor and magnificence. Foshan fancy lanterns are often decorated with copper foil paper cutting. The combination of the two makes the whole lantern more resplendent. Foshan fancy lanterns also have the characteristics of unique design, wide range of themes, diverse decorative materials, various shapes and fine workmanship. In addition, creative themes can be created according to the local characteristics of Foshan, fully reflecting the theme of South of the Five Ridges culture. In the decorative performance, Foshan fancy lantern is decorated with local folk paper-cut, with rich patterns, bright colors, exquisite beauty, magnificent and exquisitely carved atmosphere. Foshan fancy lantern has decorative beauty and reflects prominent local characteristics. In modeling, Foshan fancy lanterns are in thousands of postures and ingenious. They are good at changing and innovating regardless of colored lanterns or colored dragon. The expressive techniques are vivid and ingenious and awesome.

\section{CONCLUSION}

Foshan fancy lantern is a local folk phenomenon. It is a wonderful folk art of the South of the Five Ridges and an important part in local people's cultural life. The artistic characteristics of Foshan fancy lantern are closely related to the local culture and economy, and reflect the profound connotation of the blending of various arts. It is also a symbol of the happy value orientation and beauty in Foshan people's living customs. The crystallization of folk art created by different artistic styles is the embodiment of wisdom of Foshan people, as well as the masterpiece of high-level traditional craft.

\section{REFERENCES}

[1] Xie Zhongyuan. Research on "Productive Protection" of the National Non-material Cultural Heritage "Foshan Fancy Lantern". Journal of Foshan University (SOCIAL SCIENCE EDITION), 2013, 01: 62-67. 谢中元. 国家级非遗“佛山彩灯”的“生产性保护”研究[J].佛山科学科 技学院学报 (社会科学版)，2013，01：62-67.

[2] Peng Yikong. Foshan Fancy Lantern: Life is endless, and the lamp does not extinguish. Golden Age, 2012, 01: 63. 彭旖琨. 佛山彩灯:生不息, 灯不灭[J].黄金时代, 2012，01：63.

[3] Wang Juan. Characteristics of Foshan Paper-cut Art. Decoration, 2008, 08: 130-132. 王娟.论佛山剪纸艺术特色[J].装饰，2008，08：130132.

[4] Yao Zhonghao, Zheng Wang, Lian Xiaoxuan and Cai Zetao. Fancy Lantern Design. Science and Technology Innovation and Application, 2017, 34: 120-122. 姚仲濠, 郑旺, 连晓璇, 蔡泽涛.彩灯外观设计 [J].科技创新与应用， 2017，34 期: $120+122$

[5] Yan Mingxia. Culture Characteristics of South of the Five Ridges in Guangdong Foshan Paper-cut. Journal of Yanbian Party School, 2015, 0 : 82-85. 颜明霞. 广东佛山剪纸的岭南文化特色 [J]. 延边党校学报, 2015, 0: $82-85$. 